\title{
Evolução Temporal da Análise de Resultados do Emprego do ifR
}

\section{Temporal Evolution of the iFR (Instantaneous Wave-Free Ratio) Employment Results Analysis}

\author{
Maria Cristina Meira Ferreira ${ }^{1 \oplus}$ e Gláucia Maria Moraes de Oliveira ${ }^{2 \oplus}$ \\ Hospital Federal dos Servidores do Estado - Serviço de Hemodinâmica, ${ }^{1}$ Rio de Janeiro, RJ - Brasil \\ Universidade Federal do Rio de Janeiro, ${ }^{2}$ Rio de Janeiro, $R J-$ Brasil
}

\section{Senhor Editor,}

Lemos com muito interesse o minieditorial escrito pelos autores Chamié e Abzaid ${ }^{1}$ referente ao artigo "Avaliação de isquemia miocárdica na sala de hemodinâmica com instanataneous wave-free ratio: estudo piloto". ${ }^{2} \mathrm{O}$ minieditorial nos traduz com clareza a histórica evolução de raciocínio que devemos seguir na interpretação do estudo de fisiologia coronária na tomada de decisão terapêutica. Embora a medicina seja plena de situações binárias para resolução, como presença ou ausência de febre pelo termômetro, fica muito claro que níveis diferentes de valores referem-se a diagnósticos, prognósticos e condutas diferentes. Com relação às avaliações funcionais coronarianas, após uma enorme quantidade de estudos binários para demonstrar

\section{Palavras-chave}

Isquemia Miocárdica; Reserva Fracionada do Fluxo do Miocárdio; Stents; Doença da Artéria Coronariana; Intervenção Coronária Percutânea/métodos.

\section{Correspondência: Maria Cristina Meira Ferreira •}

Hospital Federal dos Servidores do Estado - Serviço de Hemodinâmica -

Rua Sacadura Cabral, 178. CEP 20221-903, Rio de Janeiro, RJ - Brasil

E-mail: mc.meira.ferreira@gmail.com, macris1500@gmail.com

Artigo recebido em 12/03/2020, revisado em 30/03/2020,

aceito em 30/03/2020

DOI: https://doi.org/10.36660/abc.20200195 sua validade, recentes trials citados no minieditorial nos conduzem a uma fase onde o poder decisório clínico volta a ter peso importante $e^{3}$ o dissertar desta mudança de rumos ocorreu de forma brilhante pelo minieditorial. Não deixamos de relevar o raciocínio clínico e outros fatores em nosso estudo, uma vez que o preditor de colocação de stent foi iFR $<0,87$ neste grupo, apesar do valor de corte estabelecido para o iFR ser 0,89, havendo uma significativa redução do emprego de stents.

Cabe-nos ressaltar que nosso estudo foi realizado com dados coletados no período de 2014 a 2018, abrangendo um longo período em que o iFR ainda não tinha o corte binário bem estabelecido. Até a publicação dos grandes trials Swedeheart e Define-Flair em 2017, considerava-se os valores de $\mathrm{iFR}>0,86$ e iFR $<0,93$ como zona cinzenta, e as diretrizes referentes ao método indicavam emprego do fractional flow reserve (FFR). ${ }^{4,5}$ Neste cenário de tempo, a colocação de stents em pacientes com valores de $\mathrm{iFR} \leq 0,92$ não pode ser considerada desnecessária como citada, pois havia carência de dados literários que corroborassem de forma definitiva o corte de 0,89, que só ocorreu após comparação entre os métodos FFR e iFR nos trials citados.

Agradeço a oportunidade de poder esclarecer estes pontos e corroboro que o minieditorial nos direciona e nos esclarece não só a necessidade de cada vez mais utilizarmos a fisiologia coronária, mas também de como utilizá-la nos dias de hoje, contribuindo de forma primorosa neste campo de estudo da cardiologia intervencionista.

\section{Referências}

1. Chamie D, Abzaid A. Avaliação Fisiológica Invasiva: Do Binário ao Contínuo. Arq Bras Cardiol. 2020; 114(2):265-7.

2. Vieira HCA, Ferreira MCM, Nunes LC, Cardoso CJF, Nascimento EM, Oliveira GMM. Avaliação de Isquemia Miocárdica na Sala de Hemodinâmica com iFR Instantaneous Wave-Free Ratio: Estudo Piloto. Arq Bras Cardiol. 2020; 114(2):256-64.

3. Al-Lamee R, Howard JP, Shun-Shin MJ, Thompson D, Dehbi HM, Sen S, et al. Fractional flow reserve and instantaneous wave-free ratio as predictors of the placebo-controlled response to percutaneous coronary intervention

in stable single-vessel coronary artery disease: Physiology-stratified analysis of ORBITA. Circulation. 2018;138(17):1780-92.

4. Davies JE, Sen S, Dehbi H-M, Al-Lamee R, Petraco R, Nijjer SS, et al. Use of the Instantaneous Wave-free Ratio or Fractional Flow Reserve in PCI. N Engl JMed. 2017;376(19):1824-34.

5. Götberg M, Christiansen EH, Gudmundsdottir IJI, Sandhall L, Danielewicz M, Jakobsen L, et al. Instantaneous Wave-free Ratio versus Fractional Flow Reserve to Guide PCI. N Engl J Med. 2017;376(19):1813-23. 